\title{
Laparoscopic Cystogastrostomy "Posterior Approach" for Management of Pseudo-Pancreatic Cyst: A Pilot Study
}

\author{
Abdel Gani El Shami, MD; Ahamed Mourad, MD; Tamer M Saeid Salama, MD \\ Department of General Surgery, Ain Shams University, Egypt
}

Background: Pancreatic pseudocyst is a collection of pancreatic fluid that is bounded by fibrous or granulation tissue in the lesser sac that is most often develops as a result of acute pancreatitis. This study attempted to perform the posterior laparoscopic cystogastrostomy approach for treatment of pancreatic pseudocysts and to evaluate its efficiency, feasibility and safety as a new emerging minimal invasive procedure.

Patients and methods: This was a prospective study that included 9 patients ( 4 males and 5 females) with an age range between 18 and 50 years and having symptomatic or mature $(>5 \mathrm{~cm})$ pseudo-pancreatic cysts. All patients underwent laparoscopic cystogastrostomy through posterior approach in General Surgery department at Aim Shams University hospitals.

Results: The procedure was completed through the posterior approach in 8 cases $(88.9 \%)$ and was switched to anterior approach in one case, with mean operative time $(138 \pm 27.3)$ min, mean hospital stay $(6 \pm 1.1)$ days, no mortality or major complications occurred. All the cysts were completely collapsed as detected by ultrasound within the first 2 weeks after the operation.

Conclusion: Although this was a pilot study, our results suggest that laparoscopic cystogastrostomy via the posterior approach is a safe and effective minimal invasive technique for pseudo pancreatic cysts drainage. However, the location of the cyst and the degree of adhesion between the stomach and the cyst determine the feasibility of such approach, still, more comparative studies with larger number of patients and longer duration of follow up period are recommended for better assessment and evaluation of this technique.

Key words: Laparoscopic management of cystogastrostomy, anterior approach for laparoscopic cystogastrostomy.

\section{Introduction}

Pancreatic pseudocyst is a collection of pancreatic fluid that is bounded by fibrous or granulation tissue in the lesser sac that most often develops as a result of acute pancreatitis. ${ }^{1}$ The most common cause of acute pancreatitis is gall stones (75-80\%) but some other factors such as alcohol and trauma can also act as contributors. ${ }^{2}$

Frequently, small asymptomatic pancreatic pseudocysts get better without interference and only require supportive treatment. However, medical or surgical intervention become necessary if the cysts persevere for more than 6 weeks or increase in size to become larger than $5 \mathrm{~cm}$ (mature cyst) as they become more prone to cause other complications such as infection, bleeding and obstruction. ${ }^{3,4}$

Currently, drainage of pancreatic pseudocysts is achieved by surgical, endoscopic or percutaneous interventions. However, surgery appears to be the most effective treatment as endoscopic and percutaneous drainage are associated with a high rate of recurrence and failure which can result from insufficient percutaneous drainage, occlusion or migration of the stent. ${ }^{5}$

Minimally invasive surgical techniques have greatly evolved over the past years and can be regarded as efficient and safe surgical intervention for draining pancreatic pseudocysts in comparison to open surgical drainage. ${ }^{6,7}$

Laparoscopic drainage is most frequently achieved through the anterior approach which is easier to learn but it required incision in the anterior wall of the stomach and the posterior approach which appeared as a novel and an attractive alternative as it is a highly feasible and easy to perform laparoscopic technique that has overcome the disadvantages of other drainage methods. ${ }^{8,9}$

Therefore, through this study we attempted to perform the posterior laparoscopic cystogastrostomy approach for treatment of pancreatic pseudocysts and to evaluate its efficiency, feasibility and safety as a new emerging minimal invasive procedure. 


\section{Patients and methods}

This was a prospective study that included 9 patients (4 males and 5 females) with an age ranging between 18 and 50 years and having symptomatic or mature $(>5 \mathrm{~cm})$ pseudo-pancreatic cysts. The study was conducted in the General Surgery Department at Aim Shams University hospitals during the period between July 2012 and October 2015. Approval from the ethical committee of the Faculty of Medicine at Ain Shams University was obtained to perform this study.

Patients who were selected in our study had an age range between 18 and 50 years and were diagnosed with pseudo-pancreatic cysts that were either symptomatic and non-resolving or having a size larger than $6 \mathrm{~cm}$ that were located either in the body or the tail of the pancreas and had no previous attempts for endoscopic or percutaneous drainage of the cyst (Figure 1).

Patients were excluded from the study if there was any contraindication for laparoscopic surgery or were suffering from major comorbidities such as cardiac diseases or the absence of any space between the stomach and the cyst which could be detected either in the preoperative CT or intra-operatively. Additionally, patients with pancreatic pseudocyst that were located in the head of pancreas were excluded.

All patients were evaluated by full clinical examination and a complete patient history was taken, baseline investigations were performed to all patients such as $\mathrm{CBC}$, full chemistry including $S$ amylase and liver function and coagulation profile. Pelvi-Abdominal ultrasound and multislice computerized tomography (CT) with contrast were performed for all patients to assess the site, size and nature of the cyst and its relation to the posterior wall of the stomach. ERCP was only performed for cases with suspected obstructive pancreatitis. An informed consent was taken from all patients before surgery.

The procedure was performed under general anesthesia and patients were positioned in a reverse Trendelenburg position, pneumoperitoneum was performed after inserting Veress needle with a pressure of $14 \mathrm{mmHg}$. First, a $10 \mathrm{~mm}$ camera port was inserted at the umbilicus and 3 additional ports were added under complete vision in which two $12 \mathrm{~mm}$ working ports were inserted at the right and left subcostal regions. A third $10 \mathrm{~mm}$ port was inserted at the epigastrium for liver retraction (Figure 2). Dissection of the gastrocolic ligament and the displacement of the stomach upward were performed to enter the lesser sac (Figure 3). Next, skeletonization of the cyst and adhesolysis of any adhesion between the posterior wall of the stomach and the cyst were performed. The cyst fluid was then aspirated by a spinal needle (Figure 4). Under clear visualization of the splenic vessels, a small opening in each of the anterior wall of the cyst and the posterior wall of the stomach was created (Figures 5,6). Before stapling the cyst and the stomach, full exploration and irrigation of the cyst cavity was done to ensure that the cyst was unilocular and to remove all necrotic debris and to take biopsies from any suspicious lesion under complete vision. By using $60 \mathrm{~mm}$ laparoscopic linear stapler which was inserted to the two openings, cystogastrostomy was created (Figure 7). The final opening of the pseudo cystogastrostomy was closed using vicryl 2/0 continuous sutures (Figure 8). A drain was placed near the anastomosis and a standard laparoscopic cholecystectomy was performed for patients with gall stones.

After the operation, prophylactic $3^{\text {rd }}$ generation cephalosporin was given for all patients. All patients started a liquid diet on the 2nd day after the operation. Cyst fluid aspiration and biopsies were sent for biochemical and cytological analysis and histopathology.

Patients were followed up in the outpatient clinic once every week for one month after the operation for early detection of any postoperative complications. In addition, pelviabdominal CT was done one month after the operation to confirm the complete collapse of the cyst. Follow up was then continued once every three months for one year and full clinical examination and pelviabdominal ultrasound were performed at each visit to monitor the size of the cyst.

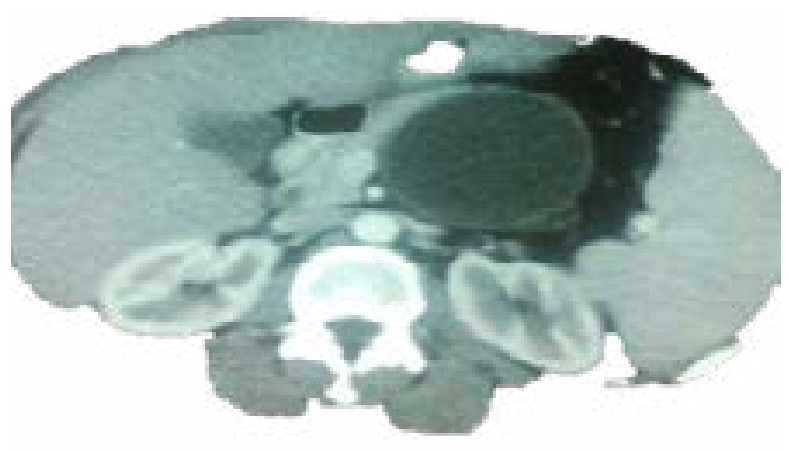

Fig 1: Abdominal C.T shows pancreatic pseudocyst. 


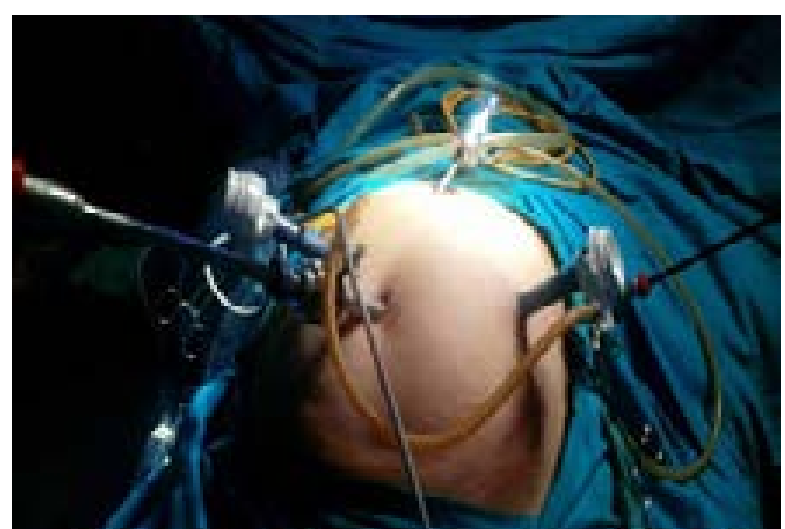

Fig 2: Picture shows ports sites.

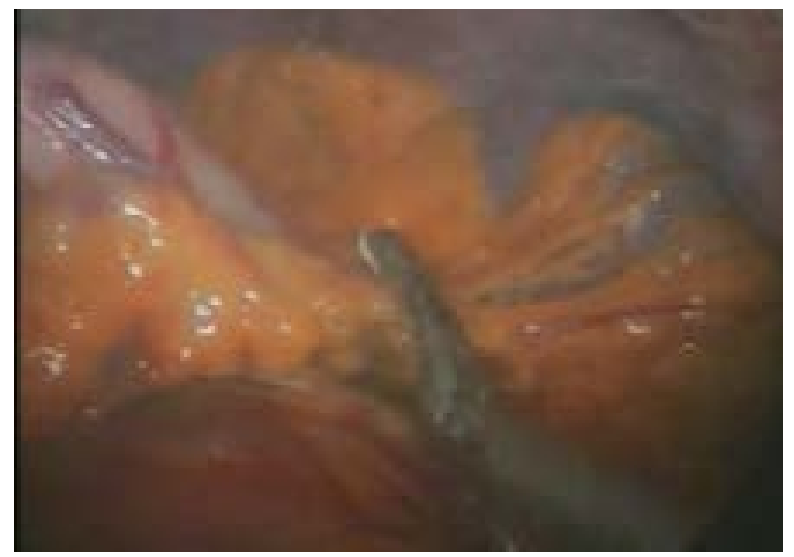

Fig 3: Dissection of gastrocolic ligament.

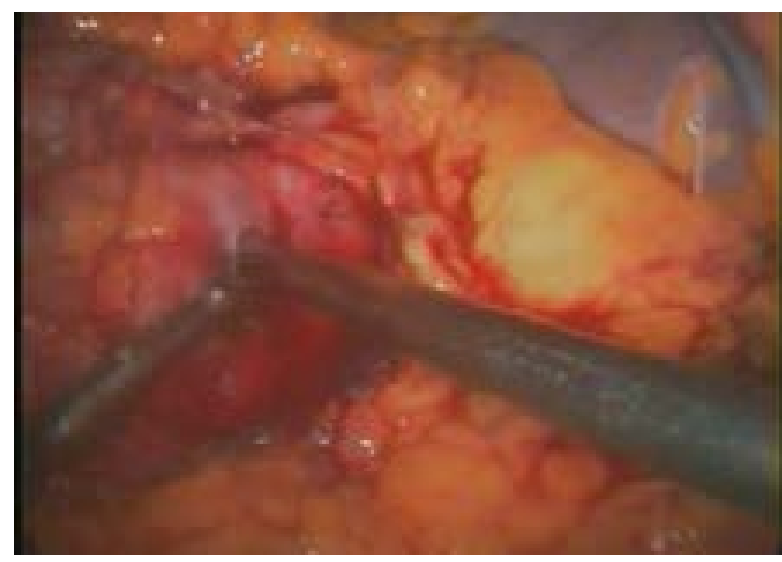

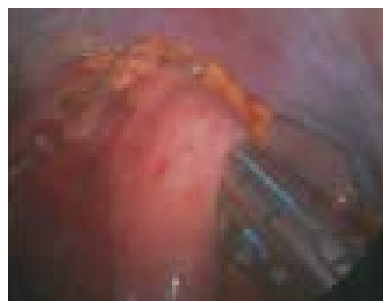

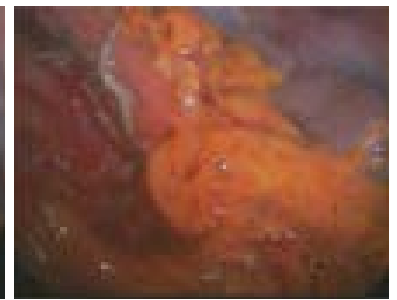

Fig 7: Cystogastrostomy is done by linear

Ftapler.
sta 7 . Cystogastrostomy is

Fig 6: Posterior gastrostomy is done by

harmonic scalpel.

Fig 5: Pseudocystomy is done by harmonic scalpel.

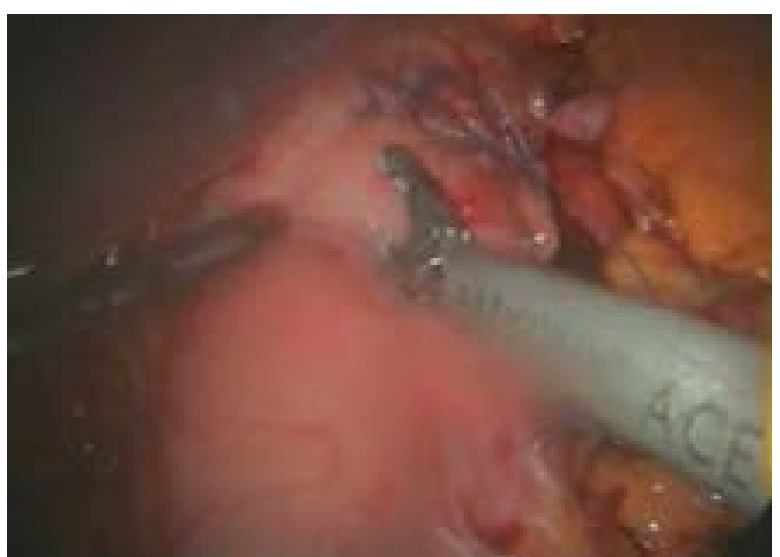

Fig 4: Aspiration of pancreatic pseudocyst fluids. 
Table 1: Patient's demographic data

\begin{tabular}{lccc}
\hline & Min & Max & Mean \\
\hline Age (year) & 18 & 50 & $41 \pm 4.59$ \\
Sex & 6 males & 3 females & - \\
Etiology & 8 biliary & 1 hyperlipidemia & - \\
Size of pseudocyst (cm) & 6 & 18 & $10 \pm 1.3$ \\
Used approach & 8 posterior & 1 anterior & $138 \pm 15.3$ \\
Operative time (min) & 90 & 180 & $6 \pm 1.1$ \\
Hospital stay (day) & 4 & 8 & \\
\hline
\end{tabular}

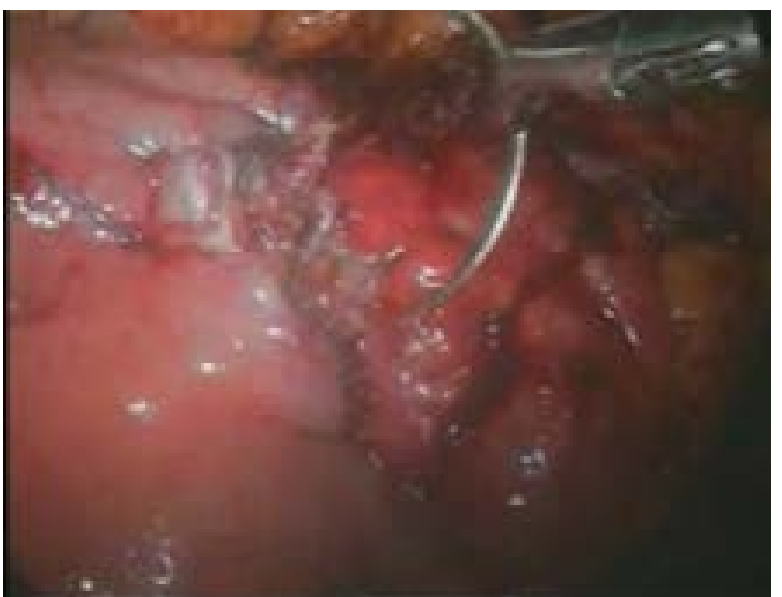

Fig 8: Completion of cystogastrostomy by intracorporeal sutures.

\section{Results}

Nine patients ( 4 males and 5 females) with a mean age of $41 \pm 4.59$ years diagnosed to have pseudopancreatic cysts with an average size of $10 \pm 1.3 \mathrm{~cm}$ were included in this study. In eight patients, pseudo pancreatic cysts were formed as a result of acute biliary pancreatitis, while in one patient it resulted from hyperlipidemia. (Table 1)

The procedure was completed through the posterior approach in 8 cases $(88.9 \%)$ and through the anterior approach in one case due to severe intraabdominal adhesions between the posterior wall of the stomach and the cyst. Cholecystectomy was performed for eight patients and all the cysts were completely collapsed as detected by ultrasound within the first 2 weeks after the operation.

The mean operative time of the procedure was $138 \pm 15.3 \mathrm{~min}$, while the mean duration of hospitalization was $6 \pm 1.1$ day. (Table 1).

All samples that were taken from the cysts showed elevated levels of Amylase, while all biopsies showed the presence of fibrotic wall rich with inflammatory cells without lining epithelium.

No mortalities or major intra or postoperative complications such as bleeding, bowel injury, anastomotic leak or recurrence were reported in this study. Minor wound infection in the port site was reported in only one case and was efficiently resolved through antibiotic treatment and frequent dressings.

\section{Discussion}

Pancreatic pseudocysts are commonly formed as a result of pancreatic inflammation where its incidence can reach $2-10 \%$ after acute pancreatitis and $10-30 \%$ after chronic pancreatitis. ${ }^{10}$ Small asymptomatic cysts are mostly resolved with supportive treatment and only require monitoring through observation and frequent radiological follow-up by ultra-sonography or CT scans. ${ }^{11}$ However, pseudocysts that persevere for more than 6 weeks or with sizes that are larger than 5 $\mathrm{cm}$ are unlikely to resolve on their own and carry the risk of other complications such as infection, bleeding or pseudoaneurism and therefore medical intervention becomes a necessity to drain such cysts. ${ }^{12,13}$

Drainage of pseudopancreatic cysts can occur externally through percutaneous aspiration and continuous catheter drainage. However, this method has a high incidence of failure (54\%) and a high rate of recurrence (63\%). ${ }^{14}$ Drainage can also take place internally through endoscopy or open or laparoscopic surgery. ${ }^{9}$

Drainage via endoscopy can be efficient but it requires highly experienced endoscopists and carries the risk of perforation and the occurrence of stent-related complications and inadequate drainage. Therefore, surgical intervention appears to be the most attractive option for drainage. ${ }^{15}$

Laparoscopic cystogastrostomy can be performed either through the stomach which is known as the 
anterior approach or through the lesser sac which is known as the posterior approach. ${ }^{16}$

In this study, 9 patients diagnosed with mature symptomatic pseudo-pancreatic cysts were operated on by laparoscopic cystogastrostomy, in which the posterior approach was performed for 8 patients, while the anterior approach was performed for only 1 patient. The mean operative time of the procedure was $138 \pm 15.3 \mathrm{~min}$ and the mean time of hospital stay was $6 \pm 1.1$ days which were highly comparable with the results obtained by Hauters $P$, et $\mathrm{al}^{17}$ who reported a mean operation time of 100 min (ranged between 60-140 $\mathrm{min}$ ) and a mean hospital stay of 6 days (ranged between 4-24 days) after laparoscopic cyst gastrostomy.

The anterior approach was performed for only 1 case due to the presence of severe adhesions between the posterior wall of the stomach and the cyst that made it difficult for the procedure to be completed through the posterior approach.

One results are in accordance with other studies $^{18,19}$ that stated that the posterior approach can be followed if there is a space or there are mild adhesions between the posterior wall of the stomach and the cyst wall. Additionally, drainage through the anterior approach is favored if the pseudocyst is adherent to the posterior wall of the stomach.

The results of all the biopsies were conclusive and have reliably confirmed our diagnosis since the biopsies were adequately obtained through the posterior approach which also allowed proper visualization of the cyst cavity. This was similarly stated in another study that reported better and more feasible visualization of the pancreatic pseudocyst via the posterior approach that allows obtaining adequate biopsies from the pseudocyst wall which helps to confirm the diagnosis and exclude other conditions such as neoplastic lesion. In contrast, the anterior approach is commonly accompanied by improper visualization which reduces the ability to obtain sufficient biopsies. Therefore, the results can be inconclusive. ${ }^{18}$

In our study, no major intra or postoperative complications such as bleeding or recurrence were reported. This was similarly described in other studies s,18,19 $^{2}$ that stated that laparoscopic cystogastrostomy through the posterior approach is more safe than the anterior approach in which anterior gastrostomy is avoided and the anastomosis is achieved by stapler and therefore better hemostasis is achieved and lower incidence of bleeding or leakage can take place. In addition, wider anastomosis is achieved in the posterior approach which reduces the risk of recurrence.

\section{Conclusion}

Although this was a pilot study, our results suggest that laparoscopic cystogastrostomy via the posterior approach is a safe and effective minimal invasive technique for pancreatic pseudocyst drainage. However, the location of the cyst and the degree of adhesion between the stomach and the cyst determine the feasibility of such approach. Still, comparative studies with anterior approach, larger number of patients and longer duration of follow up period are recommended for better assessment and evaluation of this technique.

\section{References}

1. Aljarabahm E, Ammori Bj: Laparoscopic and endoscopic approaches for drainage of pancreatic pseudocysts: A systematic review of published series. Surg Endosc 2007; 21: 1936-1944.

2. Palanivelu C, Jaypee C: Laparoscopic pancreatic surgery: Art of Laparoscopic Surgery-Textbook and Atlas First Edition 2007; 53: 851-69.

3. Palanivela C, Senthilkumar K, Madhankumar $M V$, et al: Management of pancreatic pseudocyst in the era of laparoscopic surgery experience from a tertiary center. Surg Endosc 2007; 21: 2262-2267.

4. Song S, Da-Zhi Cn, Ren Lg, et al: Aparoscopic cysto gastrostomy for treatment of pancreaticpseudo cysts a case report. World $\boldsymbol{J}$ Gastroenterol 2008; 14: 4841-4842.

5. Salih P, Kaya S, Tayfan K, et al : Case Report: Total laparoscopic cystogastrostomy for the treatment of pancreatic pseudocyst. Journal of Laparoscopic \& Advanced Surgical Techniques 2002; 12: 119-122.

6. Park A, Scwartz R: Laparoscopic pancreatic surgery. American Journal of Surg 1999; 177: 158-163.

7. Morino M, Garrone C, Locatelli C, et al: Laparoscopic management of benign pancreatic cystic lesions. Surg Endosc 1995; 9: 625.

8. Barnard B, Lance L, Michell W, et al: A comparison of anterior and posterior approaches for the surgical treatment of pancreatic pseudocyst using laparoscopic cystogastrostomy. Journal of Laparoscopic \& Advanced Surgical Techniques 2005; 15: 596600. 
9. Oida T, Kano H, Kawockn A, et al: Laparoscopic cystogostrostomy via the posterior approach for pancreatic pseudo cyst drainage. Hepatogastroenterology J 2011; 58: 110-111.

10. Obermeyer RJ, Fisher WE, SAlameh JR, et al: Laparoscopic pancreatic cystogastrotomy. Surg Laparoscopic, Endosc Percutan Tech 2003: 13: 250-253.

11. Cheruvu CV, Clarke MG, Prentice M, et al: Conservative treatment as an option in the management of pancreatic pseudocyst. Ann $\boldsymbol{R}$ Coll Surg Engl 2003; 85: 313-316.

12. Singhal D, Kakodkar R, Sud R, Chaudhary A: Issues in management of pancreatic pseudocysts. JOP 2006; 7: 502-507.

13. Warshaw AL, Rattner DW: Timing of surgical drainage for pancreatic pseudocyst. Clinical and chemical criterial. Ann Surg 1985: 202: 720-724.

14. P. K. Chowbey, Sharma A, Khullar. R: Laparoscopic intragastric stapled cystogastrostomy for pancreatic pseudocyst. Journal of Laparoscopic \& Advanced
Surgical Techniques 2002; 12: 201-205.

15. Weckman L, Kylanpaa ML, Puolakkainen $P$, et al: Endoscopic treatment of pancreatic pseudocysts. Surg Endosc 2006; 20: 603-607.

16. Cristiano C, Arice L, Dardenas $L E$, et al: Laparoscopic anagement of pancreatic pseudosysts experience at a general hospital in Mexico city. Gastroenterology de Mexico 2015; 80: 198-204.

17. Hauters P, Weerts J, Navez B. et al: Treatment of Pancreatic pseudocyst by Laparoscopic cystogastrostomy. Ann Chir 2004; 129: 347352.

18. Barragan $B$, Love $L$, Wachtel $M$, et al: A comparision of anterior and posterior approaches for the surgical treatment of pancreatic pseudocyst using laparoscopic cystogastrostomy. J Laparoendosc Adv Surg Tech 2005; 5: 596-600.

19. Kaya s, Salih P, EceK, MetinK, etal: Laparoscopic cystogastrostomy for the management of pancreatic pseudocysts. Turk J Med Sci 2008; 38: w311-317. 